\title{
Development and Validation of a Practical Machine- learning Triage Algorithm for the Detection of Patients in Need of Critical Care in the Emergency Department
}

\author{
Yecheng Liu \\ Chinese Academy of Medical Science and Peking Union Medical College \\ Jiandong Gao \\ Tsinghua University \\ Jihai Liu \\ Chinese Academy of Medical Science and Peking Union Medical College \\ Joseph Harold Walline \\ The Chinese University of Hong Kong, Prince of Wales Hospital \\ Xiaoying Liu \\ Chinese Academy of Medical Science and Peking Union Medical College \\ Ting Zhang \\ Chinese Academy of Medical Science and Peking Union Medical College \\ Ji Wu
}

Tsinghua University

Huadong Zhu ( $\nabla$ drzhuhd@sina.com )

Chinese Academy of Medical Science and Peking Union Medical College

Weiguo Zhu

Chinese Academy of Medical Science and Peking Union Medical College

\section{Research Article}

Keywords: machine-learning triage algorithm, critical care, emergency department (ED), electronic emergency triage system (EETS)

Posted Date: April 22nd, 2021

DOI: https://doi.org/10.21203/rs.3.rs-418248/v1

License: (c) (1) This work is licensed under a Creative Commons Attribution 4.0 International License. Read Full License 
Version of Record: A version of this preprint was published at Scientific Reports on December 1st, 2021. See the published version at https://doi.org/10.1038/s41598-021-03104-2. 


\section{Abstract}

Identifying critically ill patients is a key challenge in emergency department (ED) triage. Mis-triage errors are still widespread in triage systems around the world. Here, we present a machine learning system (MLS) to assist ED triage officers better recognize critically ill patients and provide a text-based explanation of the MLS recommendation. To derive the MLS, an existing dataset of 20,272 patient encounters from 2012 to 2019 from our institution's electronic emergency triage system (EETS) was used for algorithm training. We then conducted a prospective randomized cohort study of ED patients between March and April 2020. The area under the receiver operating characteristic curve (AUC) was 0.86 in a retrospective validation dataset of 2,000 randomized database cases. In the prospective cohort study, compared to the traditional triage system's $1.2 \%$ mis-triage rate, the mis-triage rate in the MLS-assisted group was $0.9 \%$. This MLS method with a real-time explanation for triage officers was able to lower the mis-triage rate of critically ill ED patients.

\section{Introduction}

Emergency department (ED) overcrowding and resulting delayed medical care are worldwide problems ${ }^{1}$ leading to increased patient mortality ${ }^{2}$. Emergency triage systems can be used to stratify patients' clinical severity and thus distribute medical resources appropriately. There are various emergency triage systems in current use around the world. The United States widely uses the Emergency Severity Index (ESI), a five-tier triage system ${ }^{3}$. At present, the People's Republic of China is using a four-tier triage system, the emergency triage scale/standard (ETS), issued by the Chinese National Ministry of Health in $2011^{4}$. Very much like in the ESI, patients triaged into ETS levels 1 or 2 will likely need critical care and are sent directly to a resuscitation room. Patients triaged into ETS levels 3 or 4 will be considered nonemergent and will have to wait to see a consulting physician. The ETS triage system is generally accurate and useful in most cases ${ }^{5}$. However, there are still some critical patients triaged into ETS levels 3 or 4 that need to wait in line with other non-critical patients until they are identified in subsequent medical encounters, sometimes several hours after the initial triage screening. For these patients, waiting for medical attention can be quite dangerous. Unfortunately, this type of life-threatening mis-triage can be found in every triage system in the world ${ }^{6}$.

Currently, triage is mostly performed by experienced nurses based on a mix of subjective (e.g., a patient's level of distress) and objective (e.g., vital signs) criteria. There will be some pre-established 'red-flag' values for these criteria, such as a systolic blood pressure below $90 \mathrm{mmHg}$, loss of consciousness, or complaints of chest pain, etc. However, there are some patients whose vital signs or medical histories do not cross these thresholds, and further improvements to the identification of critical patients by considering patient age, sex, multiple vital signs, ED arrival mode, etc. may be possible. Artificial intelligence approaches may have advantages in such complex, non-linear situations ${ }^{7}$.

This study aimed to derive and then validate a machine learning method to support the identification of potentially life-threatening mis-triage and offer at triage a real-time, detailed explanation showing why the 
algorithm scored a patient as high risk in the hope of improving the detection of ED patients in need of critical care.

\section{Methods}

We used CatBoost Python package (open-source, Russia) as a training model for the prediction of mistriage ${ }^{8 ;} ;{ }^{9}$. We implemented a pre-science-like approach ${ }^{10}$ in predicting whether patients were going to be a "life-threatening mis-triage" case. This approach can give a patient's overall relative risk of such mistriage as well as the relative risk features of the case leading to the MLS protocol's positive call to aid ED staff (see Figure S1).

We analyzed the ETS database from the ED of a major tertiary-care urban teaching hospital for this study. An electronic ETS (EETS) classification started being used in $2012^{11}$. The ED triage staff inputs basic information about patients into the EETS to arrive at the "traditional" ETS emergency triage level classifications. EETS uses the four-tier ETS algorithm: immediate (level 1), emergent (level 2), urgent (level 3), and non-urgent (level 4). EETS also collects patient age, sex, arrival mode, arrival time, triage vital signs (pulse rate, systolic and diastolic blood pressure, respiratory rate, temperature, and oxygen saturation), level of consciousness, chief complaint(s), blood sugar level and visual analogue (pain) scale (VAS). EETS data from November 2012 to December 2019 were available for analysis. We excluded patients who were dead on ED arrival, or who left before being triaged. During feature engineering, we excluded those data categories with $>50 \%$ missing information or clearly spurious data (systolic blood pressure $>300 \mathrm{mmHg}$, diastolic blood pressure $>200 \mathrm{mmHg}$, pulse rate $>300 / \mathrm{min}$, or oxygen saturation $>$ $100 \%)$. The ED resuscitation room was the site of monitoring for patients who needed critical care or immediate treatment. Final diagnosis data was collected using the International Classification of Diseases, Tenth Version (ICD-10) codes for each patient. What major treatments were performed in the resuscitation room were also noted, including ventilator use, vasoactive drug use, or major (invasive) procedures. Resuscitation room mortality was also recorded.

Based on eight years of retrospective data, our machine learning system was designed to identify "lifethreatening mis-triaged patients", defined as those patients who were initially triaged as level 3 or 4 by EETS, but were then admitted to the resuscitation room within 24 hours. In the development phase of our proposed MLS, we used all positive 'life-threatening mis-triaged' cases from the dataset, while negative cases were randomly selected from the same dataset. After training and validation, the MLS first outputs a relative risk (Odds ratio in log(e) space) of whether a patient should be admitted in resuscitation room immediately. In addition, the MLS also outputs the relative risk of every single feature collected at triage, so the medical staff can know why the patient should be admitted to the resuscitation room.

In the prospective cohort design, we randomly selected 30 days between March 1 and April 29, 2020 as a control group using the standard EETS protocol, while in the remaining 30 days between March 1 and April 29, 2020, our MLS protocol was adopted as an intervention group. During the prospective cohort phase, in the intervention group, for better use of the MLS, we provided an individual's odds ratios (ORs) 
to help further triage (an example is shown in Fig. 1). The study was approved by the ethics committee of Peking Union Medical College Hospital prior to the study commencing.

\section{Results}

Between November 1, 2012 to December 31, 2019, there were 1,023,613 ED visits, for which the EETS triage-level information is shown in Table 1. The 'life-threatening mis-triage' rate in this group was $1.12 \pm$ $0.04 \%$. Most of the 'life-threatening mis-triage' cases(76.18\%) admitted to the resuscitation room within 4 hours after triage. The top 20 final diagnoses of patients entering the ED resuscitation room are shown in Table 2. The average rate of ventilator use for patients in the resuscitation room was $29.6 \%$, vasoactive drug use was $30.2 \%$, invasive procedure rate was $32.5 \%$, and resuscitation room mortality was $8.6 \%$.

Table 1

Patient characteristics

\begin{tabular}{|llll|}
\hline Characteristics & Development set & Validation set & $\begin{array}{l}\text { Prospective test } \\
\text { set }\end{array}$ \\
\hline Years-months & $\begin{array}{l}2012.10- \\
2019.12\end{array}$ & $\begin{array}{l}2012.10- \\
2019.12\end{array}$ & $\begin{array}{l}2020.03- \\
2020.04\end{array}$ \\
\hline Total no. of cases & 20,272 & 2,000 & 17,072 \\
\hline $\begin{array}{l}\text { Age, years: median (25th, 75th } \\
\text { percentiles) }\end{array}$ & $53.0(34.0,67.0)$ & $54.0(35.0,68.0)$ & $51.0(34.0,65.0)$ \\
\hline Female (\%) & $55.4 \%$ & $54.4 \%$ & $53.9 \%$ \\
\hline ETS level 3 (\%) & $48.7 \%$ & $51.3 \%$ & $47.7 \%$ \\
\hline Systolic blood pressure & $122.0(109.0$, & $122.0(109.0$, & $125.0(113.0$, \\
\hline Diastolic blood pressure & $138.0)$ & $139.0)$ & $140.0)$ \\
\hline Heart rate & $75.0(66.0,84.0)$ & $74.0(65.0,85.0)$ & $77.0(68.0,86.0)$ \\
\hline Oxygen saturation & $88.0(76.0,102.0)$ & $89.0(76.0,103.0)$ & $89.0(78.0,102.0)$ \\
\hline Shock index & $99.0(97.0,100.0)$ & $98.0(97.0,100.0)$ & $99.0(97.0,100.0)$ \\
\hline Pulse pressure & $0.7(0.6,0.9)$ & $0.7(0.6,0.9)$ & $0.7(0.6,0.8)$ \\
\hline Altered mental status & $46.0(36.0,60.0)$ & $46.0(36.0,60.0)$ & $47.0(38.0,60.0)$ \\
\hline Arrival mode & $88(0.4 \%)$ & $14(0.7 \%)$ & $21(0.1 \%)$ \\
\hline Ambulance & & & $647(3.8 \%)$ \\
\hline Non-Ambulance & $3,100(14.3 \%)$ & $455(22.8 \%)$ & $15,518(96.2 \%)$ \\
\hline & $17,172(85.7 \%)$ & $1,545(77.2 \%)$ & \\
\hline
\end{tabular}


Table 2

The top twenty final diagnoses for patients entering the resuscitation room

\begin{tabular}{|l|l|}
\hline Diagnostic category & Proportion \\
\hline Acute coronary syndrome & $9.4 \%$ \\
\hline Acute cerebrovascular disease & $7.8 \%$ \\
\hline Abdominal pain & $6.9 \%$ \\
\hline Pneumonia & $6.5 \%$ \\
\hline Gastrointestinal hemorrhage & $5.3 \%$ \\
\hline Other lower respiratory diseases & $5.2 \%$ \\
\hline Shock & $4.7 \%$ \\
\hline Congestive heart failure & $3.9 \%$ \\
\hline Cardiac dysrhythmias & $2.8 \%$ \\
\hline Syncope & $2.7 \%$ \\
\hline Trauma & $2.5 \%$ \\
\hline Diabetes mellitus with complications & $2.3 \%$ \\
\hline Respiratory failure & $2.1 \%$ \\
\hline Sudden death & $1.9 \%$ \\
\hline Septicemia & $1.8 \%$ \\
\hline Fluid and electrolyte disorders & $1.6 \%$ \\
\hline Chronic obstructive pulmonary disease & $1.6 \%$ \\
\hline Fever of unknown origin & $1.5 \%$ \\
\hline Non-specific chest pain & $1.4 \%$ \\
\hline Poisoning & $1.3 \%$ \\
\hline
\end{tabular}

After data pre-processing (see Fig. 2), 22,272 cases were included for MLS training and validation, including 7,382 positive cases and 14,890 negative cases (see Table 1). In the prospective testing phase (March 1 to April 31, 2020) 17,073 cases were included.

\section{MLS Performance}

The MLS protocol achieved an AUC of 0.86 on the retrospective validation dataset (see Figure S2). The baseline 'life-threatening mis-triage' rate in the prospective control group was $1.2 \%$ and $0.9 \%$ in the 
intervention group, while the number of times a triage nurse called a supervising ED physician for aid in a triage decision increased $24.3 \%$ in the intervention group compared to the control group.

Looking more deeply into the MLS model, we used SHAP ${ }^{12}$ (SHapley Additive exPlanations), a game theoretic approach to explain the output of an MLS model based on the training datasets. We used the SHAP approach to delineate the effect of important features for groups and individuals to the overall MLS algorithm. The SHAP results are shown Fig. 3 and the Pearson correlation of numerical features and labels is shown in Figure S3.

This MLS model integrates arrival mode, age, sex, and arrival time into the system, none of which are included in the current emergency triage standard. According to our results, arrival mode, age, and sex were the top-three most important features for the MLS protocol.

We also found that higher or lower heart rates, extreme diastolic or systolic pressures, lower oxygen saturation, older age, and arrival at night (Figure S4-S9) were associated with a higher mis-triage risk. In determining whether a triage Level 3 or 4 patient needs critical care, the shock index and pulse pressure were important triage features both to the MLS and to traditional triage (see Figure S10 and Figure S11). In further analysis, shock index has a higher sensitivity in older patients while being less sensitive in younger patients (see Figure S12). On the other hand, pulse pressure has a higher sensitivity in younger patients while being less sensitive in older patients (see Figure S13).

\section{Discussion}

Based on 1,023,613 visits, we built an MLS protocol with a satisfactory AUC to test for the odds of a patient with an initial ETS Level 3 or 4 encountering a 'life-threatening mis-triage' situation and requiring up-triage into the resuscitation room during their ED stay. At the same time, we used interpretability as an essential practical reference-point for triage officers, so that medical staff may intuitively understand the weight of each triage feature in the conclusions given by the MLS. By being more 'understandable' than a simple 'black box' system, we hoped this MLS would better aid in making triage decisions. Used in a prospective dataset, we found a significant decrease in the 'life-threatening mis-triage' rate, demonstrating its effectiveness and usefulness.

Current clinical triage systems, including the ESI in the United States and the ETS in China, have significant limitations. They rely to some degree on the subjective judgment of triage staff, which has led to significant variability in triage results ${ }^{13}$, especially in pediatric patients ${ }^{14}$. About half of the ED patients in the United States are triaged as ESI Level $3^{15}$, and many of these patients have conditions that potentially are quite emergent ${ }^{16}$. The mortality rate of ESI level 3 patients in most triage systems is not low, even reaching nearly half of that of level 2 patients ${ }^{6}$. In China, most patients are triaged into ETS Level 3 or $4^{5}$. In our retrospective study, the rate of 'life-threatening mis-triage' in ED patients with initial ETS Level 3 or 4 was $1.12 \%$. Reducing this rate may save lives. For a typical ED with 100,000 patient 
visits a year, reducing the mis-triage rate to $0.9 \%$ with an MLS as we were able to do in this study, would translate into hundreds of fewer mis-triage cases.

Machine learning can modify its response patterns by creating data systems, developing algorithms, and applying them to inference outcomes from new data, which is most applicable to a number of complex, nonlinear relationships. Machine learning models have been applied to predict outcomes in different medical fields, such as predicting hypoxemia during surgery ${ }^{10}$, predicting mortality in patients with sepsis ${ }^{17}$, acute cardiac complications in patients with acute chest pain ${ }^{18}$, and the likelihood of severity and hospitalization in children ${ }^{19}$ and adults ${ }^{20}$ with asthma or COPD exacerbations, respectively. Previous studies have also looked at adult ED triage to predict patients' risk of critical illness or hospitalization $21 ; 22$, but these studies did not provide any explanatory interpretation on the clinical side, making it difficult for MLS results to be accepted by medical staff and integrated into clinical practice. Although relatively straightforward, providing the MLS reasoning allowed us to prospectively validate this MLS protocol and directly test its utility on 'real-life' triage cases. Compared to previous studies, our approach provides a detailed indication of a patient's risk of requiring critical care. In our study's MLS protocol, each patient is given an individualized odds ratio with the specific weight of each triage feature used in artificial intelligence decision making listed and displayed. Such a descriptive read-out provides a triage nurse with additional material to confidently call a supervising ED physician to assess a patient's condition. In our prospective results, it increased physician consults to tirage by almost $25 \%$ and reduced the 'life-threatening mis-triage' rate to less than one percent. Traditionally, because of the high workload of ED nurses and physicians, unless a patient's condition differed significantly from the electronic grading system, triage nurses were reluctant to call a physician for consultation. Our MLS can give triage nurses more confidence to make such a call.

Machine learning has some unique advantages in triage. Factors can now be included that were traditionally left out of formal triage protocols. For example, a patient's time of arrival are important ${ }^{23}$, and a patient's mode of arrival is also anecdotally acknowledged as being important by experienced triage nurses: patients that arrive on ambulances or arrive at night tend to be sicker. In fact, in our MLS results, the most important triage feature was arrival mode, which is similar to other machine learning triage studies ${ }^{21}$. Age is also an essential parameter in most critical care scoring systems, as older patients often have more underlying diseases and poorer prognoses than younger patients ${ }^{24}$. However, age cutoffs in traditional triage systems tend to be too blunt. For example, the risk profile of a 64 -year-old compared to a 65 -year-old is quite similar, despite a cutoff at $65^{25}$. An MLS protocol that uses age as a continuous variable rather than simple cutoffs may provide better predictive power for 'edge' cases. Similarly, in the traditional triage system, it is often exhausting to determine the normal value and critical value of heart rate for pediatric patients of different ages. On the other hand, machine learning triage systems can computationally combine a patient's age with heart rate and other indicators to quickly give an appropriate weighting. Likewise, sex is not used as a high-risk factor in traditional triage, but there are differences between males and females in the risk-profiles of many diseases, and a machine learning 
method can consider a patient's sex in formulating a risk assessment. In our study, sex was one of the top three most important triage characteristics.

We included two additional indicators in our study: pulse pressure and shock index, which are two circulation indicators that have been found to be highly correlated with acute illness severity in previous studies ${ }^{26-29}$ but have not been widely used in emergency triage to date. Our results found these two indicators to be beneficial as emergency triage characteristics, even more important than systolic blood pressure. Interestingly, we found pulse pressure has a lower sensitivity in older patients, which has not been reported before, perhaps because older patients have decreased vascular compliance, making their pulse pressure higher, thus lowering its sensitivity. The situation with shock index was just the opposite, as it has a higher sensitivity in older patients. This may be due to older patients' relatively poor cardiac compensation ability, requiring a higher heart rate to compensate for any decrease in blood pressure.

We should note that some important traditional triage factors were not included in our MLS system, specifically respiratory rate, which was not included due to many missing data values in the dataset, suggesting poor nurse compliance in obtaining this feature. Respiratory rate measurement has been criticized as requiring time and skills for an accurate measurement ${ }^{30}$. Missing data values for temperature were also large, possibly because most patients had a normal temperature or did not require a routine temperature check, and possibly because more 'traditional' temperature measurements are more invasive and time-consuming for triage staff. Chief complaint is clearly important but was also not included because of the large variability in complaints and many missing values. Chief complaints are unfortunately quite subjective, and future MLS acquisition and standardization techniques using very large datasets will likely be needed to better include this as a viable triage factor in the future. All these missing data values suggest that the EETS system may need to change, and some threshold indicators (e.g., shortness of breath, ischemic chest pain, etc.) can possibly be used instead. Interestingly, the noninclusion of these indicators did not affect the validity and usefulness of this study's results. On the contrary, the results of this study are more generalizable because we only used easily obtainable, objective indicators.

\section{Limitations}

Our study has a few limitations. Some features we used in the model such as arrival mode, arrival time, and initial emergency triage level can be influenced by local system factors in different geographic areas that use different emergency triage standards. However, MLS can use data from different hospitals to learn quickly and update, thus better meeting the needs of specific hospitals. Secondly, our prospective validation dataset only had two months (March and April 2020) of data to evaluate. We plan to continue to examine the MLS results in different seasons in the future. Thirdly, we only focused on patients triaged to Levels 3 and 4 in this study, focused on finding 'under-triaged' patients in this group. Future work can examine rates of 'over-triage' in Levels 1 and 2. Lastly, although we found that many factors were important for identifying critically ill patients, because of missing data, we were unable to include respiratory rate, temperature, or chief complaint in the system. As noted earlier, this either means better 
collection methods are needed for these factors or these factors are best not included as either the lack of uptake by triage staff or their subjectivity make their use in an MLS system unreliable.

\section{Conclusions}

It is challenging to accurately differentiate critically ill from stable patients in the ED, which can lead to life-threatening cases of mis-triage. This study's MLS was able to reduce the life-threatening mis-triage rate from $1.2-0.9 \%$ in the prospective arm. Compared to previous studies, our MLS approach also provided a detailed explanation of each patient's risk of mis-triage, and caused nearly $25 \%$ more triage calls to supervising ED physicians. Although MLS protocols can lower mis-triage rates, future prospective studies may shed more light on potential reductions in patient morbidity and mortality.

\section{Declarations}

\section{Acknowledgements}

We would like to thank Xu Han and Zexi Li for their contributions detailing clinical information.

\section{Author contributions}

We confirm that there is no overlap with previous publications, and we confirm that the manuscript, including related data, figures, and tables, has not been published previously and that the manuscript is not under consideration elsewhere. We also confirm that all authors have made substantial contributions to all of the following: 区the conception and design of the study, or acquisition of data, or analysis and interpretation of data, \drafting the article or revising it critically for important intellectual content, $\otimes$ final approval of the version to be submitted.

\section{Statement}

The authors declare that all methods in the article were carried out in accordance with relevant guidelines and regulations.

\section{Competing interests statement}

The authors have no financial or other conflicts of interest regarding this article.

\section{Ethical approval}

We have written informed consent obtained from all participants.

\section{Funding}

Supported by the Non-Profit Central Research Institute Fund of Chinese Academy of Medical Sciences (Grant Number: 2019XK320035). 
Supported by the National Key Research \& Development Program of China (Grant Number: 2018YFC0116800).

Supported by the Beijing Municipal Natural Science Foundation (Grant Number: L192026).

\section{References}

1. Khalifa, M. \& Zabani, I. Reducing Emergency Department Crowding: Evidence Based Strategies. Stud Health Technol Inform. 226, 67-70 (2016).

2. Sun, B. C. et al. Effect of Emergency Department Crowding On Outcomes of Admitted Patients. Ann. Emerg. Med. 61, 605-611 (2013).

3. McHugh, M., Tanabe, P., McClelland, M. \& Khare, R. K. More Patients are Triaged Using the Emergency Severity Index than any Other Triage Acuity System in the United States. Acad. Emerg. Med. 19, 106109 (2012).

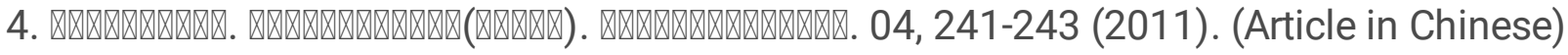

5. Hunag Xueli囚et al. Reliability and validity of emergency triage standards. Chinese Journal of Nursing. 51, 365-369 (2016).

6. Farrohknia, N. et al. Emergency Department Triage Scales and their Components: A Systematic Review of the Scientific Evidence. Scand J Trauma Resusc Emerg Med. 19, 42 (2011).

7. Shafaf, N. \& Malek, H. Applications of Machine Learning Approaches in Emergency Medicine; A Review Article. Arch Acad Emerg Med. 7, 34 (2019).

8. Lundberg, S. M. et al. From Local Explanations to Global Understanding with Explainable Al for Trees. Nat Mach Intell. 2, 56-67 (2020).

9. Mistry, B. et al. Accuracy and Reliability of Emergency Department Triage Using the Emergency Severity Index: An International Multicenter Assessment. Ann. Emerg. Med. 71, 581-587 (2018).

10. Travers, D. A., Waller, A. E., Katznelson, J. \& Agans, R. Reliability and Validity of the Emergency Severity Index for Pediatric Triage. Acad. Emerg. Med. 16, 843-849 (2009).

11. Christ, M., Grossmann, F., Winter, D., Bingisser, R. \& Platz, E. Modern Triage in the Emergency Department. Dtsch. Arztebl. Int. 107, 892-898 (2010).

12. Dugas, A. F. et al. An Electronic Emergency Triage System to Improve Patient Distribution by Critical Outcomes. J. Emerg. Med. 50, 910-918 (2016).

13. Lundberg, S. M. et al. Explainable Machine-Learning Predictions for the Prevention of Hypoxaemia During Surgery. Nat Biomed Eng. 2, 749-760 (2018).

14. Taylor, R. A. et al. Prediction of In-Hospital Mortality in Emergency Department Patients with Sepsis: A Local Big Data-Driven, Machine Learning Approach. Acad. Emerg. Med. 23, 269-278 (2016).

15. Liu, N. et al. Risk Scoring for Prediction of Acute Cardiac Complications From Imbalanced Clinical Data. IEEE J Biomed Health Inform. 18, 1894-1902 (2014). 
16. Goto, T., Camargo, C. J., Faridi, M. K., Freishtat, R. J. \& Hasegawa, K. Machine Learning-Based Prediction of Clinical Outcomes for Children During Emergency Department Triage. JAMA Netw Open. 2, e186937 (2019).

17. Goto, T., Camargo, C. J., Faridi, M. K., Yun, B. J. \& Hasegawa, K. Machine Learning Approaches for Predicting Disposition of Asthma and COPD Exacerbations in the ED. Am. J. Emerg. Med. 36, 16501654 (2018).

18. Raita, Y. et al. Emergency Department Triage Prediction of Clinical Outcomes Using Machine Learning Models. Crit. Care. 23, 64 (2019).

19. Levin, S. et al. Machine-Learning-Based Electronic Triage More Accurately Differentiates Patients with Respect to Clinical Outcomes Compared with the Emergency Severity Index. Ann. Emerg. Med. 71, 565-574 (2018).

20. Lin, P., Shi, F., Wang, L. \& Liang, Z. A. Nighttime is Associated with Decreased Survival for Out of Hospital Cardiac Arrests: A Meta-Analysis of Observational Studies. Am. J. Emerg. Med. 37, 524-529 (2019).

21. Vercelli, M. et al. Age-Related Mortality Trends in Italy From 1901 to 2008. PLoS One. 9, e114027 (2014).

22. Jylhävä, J., Pedersen, N. L. \& Hägg, S. Biological Age Predictors. EBioMedicine. 21, 29-36 (2017).

23. Zhu, C. S. et al. Shock Index and Pulse Pressure as Triggers for Massive Transfusion. J Trauma Acute Care Surg. 87, S159-S164 (2019).

24. El, A. A. et al. Vital Sign Prediction of Adverse Maternal Outcomes in Women with Hypovolemic Shock: The Role of Shock Index. PLoS One. 11, e148729 (2016).

25. Campos-Serra, A. et al. The Use of the Shock Index as a Predictor of Active Bleeding in Trauma Patients. Cir Esp. 96, 494-500 (2018).

26. Franklin, S. S. \& Wong, N. D. Pulse Pressure: How Valuable as a Diagnostic and Therapeutic Tool? J. Am. Coll. Cardiol. 67, 404-406 (2016).

27. Simoes, E. A., Roark, R., Berman, S., Esler, L. L. \& Murphy, J. Respiratory Rate: Measurement of Variability Over Time and Accuracy at Different Counting Periods. Arch. Dis. Child. 66, 1199-1203 (1991).

28. Dorogush, A. et al. Fighting Biases with Dynamic Boosting. (2017).

29. Anna Veronika Dorogush, Vasily Ershov, Andrey Gulin "CatBoost: gradient boosting with categorical features support". Workshop on ML Systems at NIPS 2017. Available at: https://github.com/catboost/catboost.

30. Liu Jihai, et al囚Emergency triage system design and implementation based on cognitive task analysis. Chin J Crit Care Med 4, 224-232 (2013).

\section{Figures}




\section{Figure 1}

Overall Estimated relative risk 2.252 (OR=9.51 in log(e) space) $f(x)=2.252$

$55=$ diastolic blood pressure

83 = systolic blood pressure

$1.084=$ shock index

$23=$ arrival time

$$
M=\operatorname{sex}
$$

walk in = arrival mode

$$
58=\text { age }
$$

$98=$ oxygen saturation

$28=$ pulse pressure

3 other features

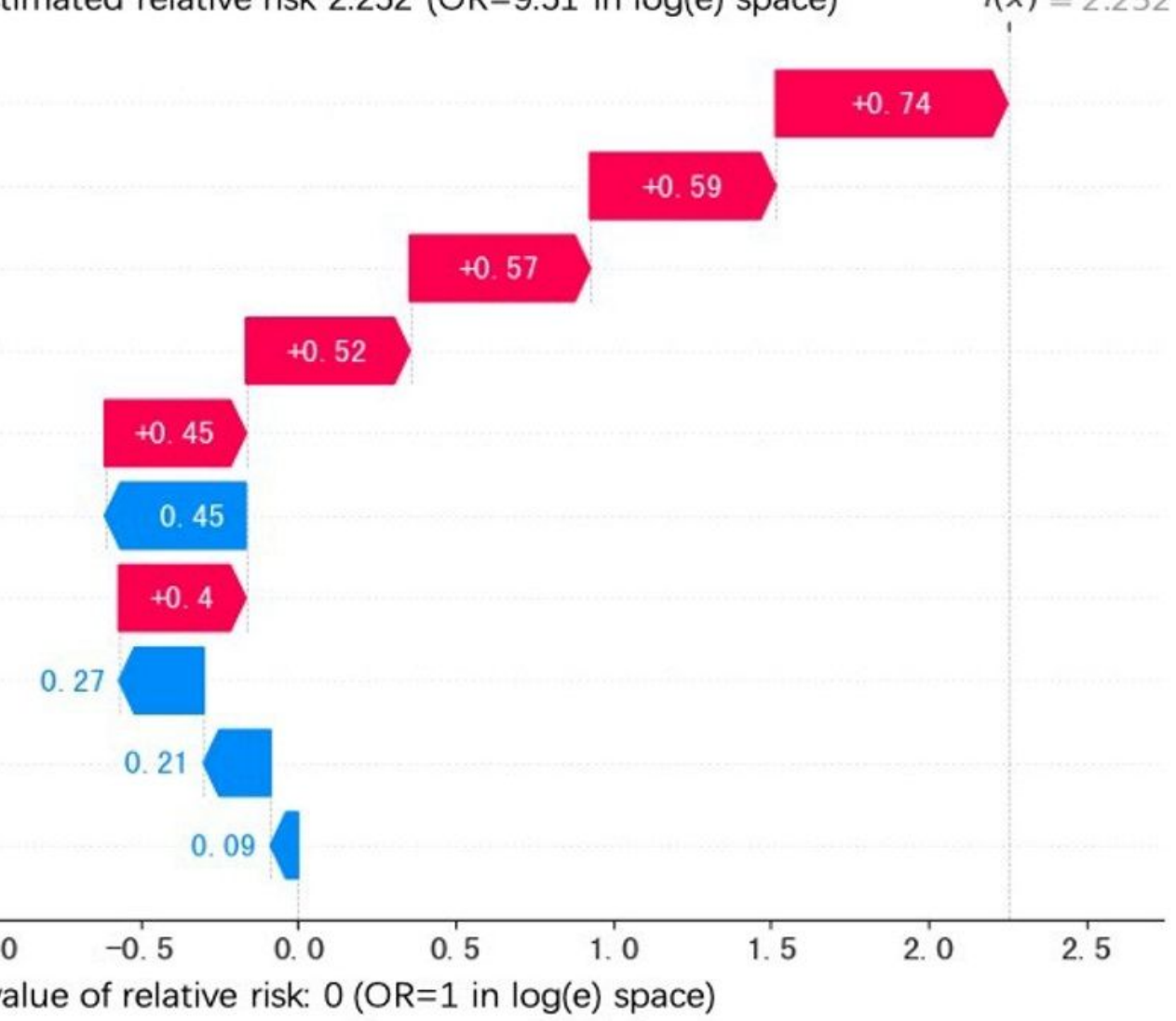

\section{Figure 1}

An example of an individual's relative risk results. 
Figure 2

\section{DATASET}

16 variables and 1,023,613 records

\section{Sampling}

16 variables and 22,272 records

Feature engineering

- Shock index

- Triage time (Nth hour)

- Pulse pressure

19 variables and 22,272 records

Variables removal

- ID

- Triage datetime

- >50\% Nans:

- Blood sugar

- Body temperature

- VAS

- Respiratory rate

- Chief complaint

12 variables and 22,272 records

PREPROCESSED DATASET

12 variables ( 1 outcome) and 22,272 records

Figure 2

Flow chart of data processing during the study. 


\section{Figure 3}

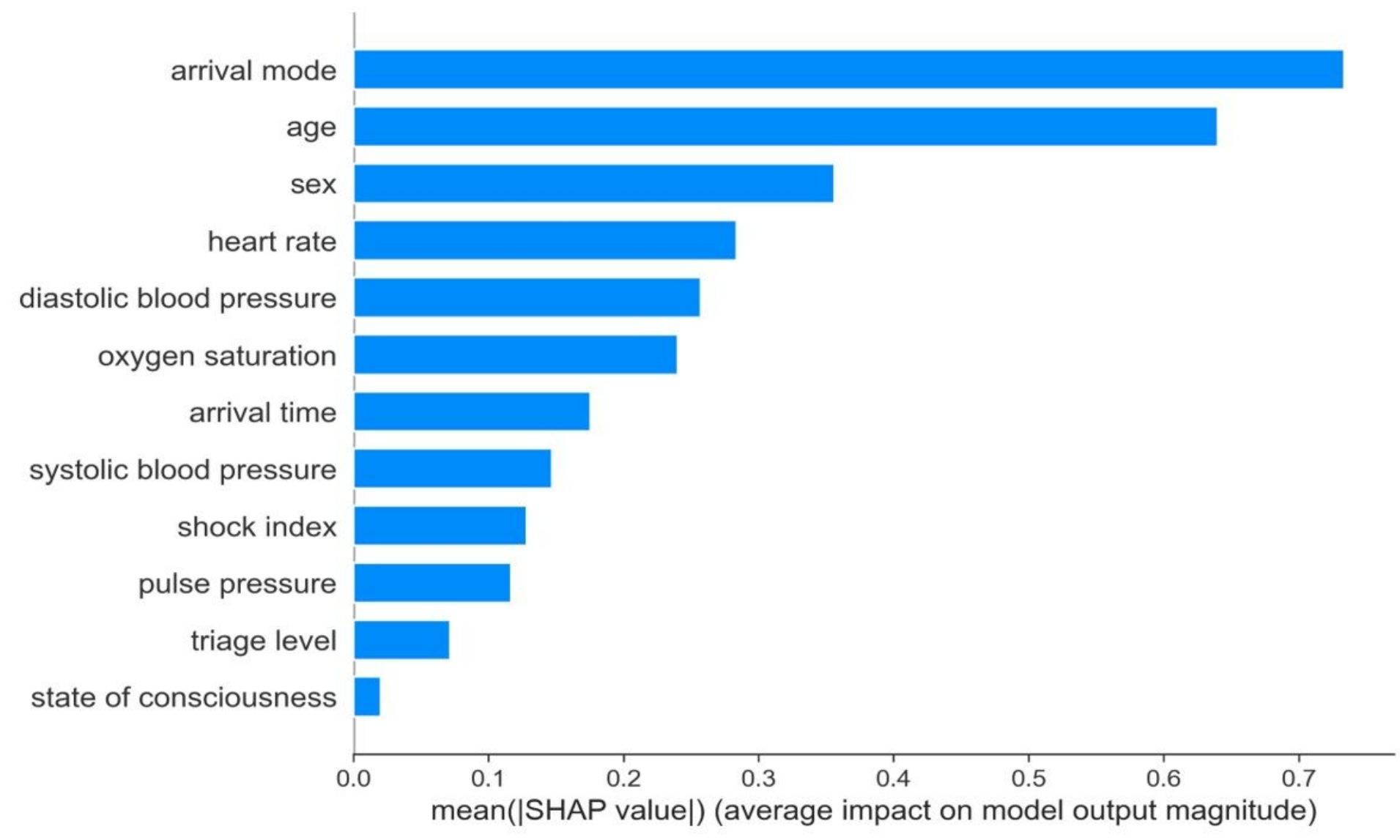

\section{Figure 3}

The impact of each model element to the overall model.

\section{Supplementary Files}

This is a list of supplementary files associated with this preprint. Click to download.

- figures1.tif

- figures2.tif

- figures3.tif

- figures4.tif

- figures5.tif

- figures6.tif

- figures7.tif

- figures8.tif

- figures9.tif

- figures10.tif 
- figures11.tif

- figures12.tif

- figures13.tif

- figures14.tif 